\title{
An Electricity Pricing Oriented Memory Calculation Hybrid Application Mode
}

\author{
Haohan $\mathrm{Hu}^{1, \mathrm{a}}$, Chen $\mathrm{Qi}^{1, \mathrm{~b}}$ and Jinglin Guo ${ }^{1, \mathrm{c}}$ \\ ${ }^{1}$ Beijing China Power Information Technology Co.,Ltd.,State Grid Electric Power Research \\ Institute,Haidian District,Beijing 100192,China \\ aprojectguo@126.com, b13801183551@139.com, cguojinglin@sgitg.sgcc.com.cn
}

Keywords: smart power, pricing, memory calculation, infrastructure.

\begin{abstract}
We discuss the key technology of smart power, analyze the technological features of big data technology and the column type database and present the basic computing architecture that fit for OLAP and OLTP hybrid mode. We provide an architecture solution based on the $\mathrm{x} 86$ server and open source components. We finally propose the evolution plan of this infrastructure based on PCI-E data exchange technology.
\end{abstract}

\section{Introduction}

With the development of user selling electricity market, smart power will become the trend. The feature of smart power is orderly electricity and gradually achieving 'peak load shifting', and the key to realize it is the rate control technology of smart meters. In 2015, the number of Watt-hour meter users approximates to 250 million in China, and half of them use smart meters which will popularize rapidly. In terms of the industrial and population distribution in China, more than 10 provinces will hold the numbers of installed smart meters exceeding 10 million. Orderly electricity needs to be guided by price, and the pricing process is a typical OLTP and OLAP hybrid application. Users buying electricity belongs to OLTP while smart meters pricing belongs to OLAP, and both of them share fundamental data.

Power meter pricing is to measure all types of electricity quantity including meters reading, transformer loss and line loss power, and calculate the electricity price according to time-sharing electricity prices. If we use every 15 seconds as the billing cycle of smart power meters, it will produce large amount of computation and data, which may become a big challenge for implementation.

\section{Research Status.}

For large OLTP and OLAP hybrid applications, people generally choose column type database to implement it and use memory databases and big data analysis technology in this process. Column storage is a new database read/write mode discriminating from the traditional row storage. The $\mathrm{read} / \mathrm{write}$ mode of traditional database is based on row and executes from the first column to the last column. However, the writing operation of row storage which is fit for structural data is completed once for all, which may get a low efficiency for large data. Column storage changes this mode completely. It splits one row as a single column to preserve before writing, which satisfies the demand of large data storage as a scattered data preservation mode. For data reading, row storage reads the entire row of data, which will produce lots of redundant data and lead to serious bandwidth occupation problems, but column storage can well avoid these problems.

At present, the main commercial big data analysis systems mostly adopt column storage, such as ORACLE Exadata machine, HANA of SAP. In 2012, HP announced and introduced a new enhanced information optimization solution, which came from the two companies Vertica and Autonomy acquired in 2011. Vertica is a column technology company, and the data storage mode is based on column storage technology. In addition, as one of the earliest manufactures engaged in the development of column type database, Sybase introduced the column type database Sybase IQ 15.4 which integrated Hadoop and MapReduce. 
Memory database is widely applied in huge data calculation, and there are cases in commercial use and open source technology ${ }^{[1,2]}$. For example, Oracle introduced an in-memory database option. Oracle in-memory database option supports two kinds of data storage format, namely traditional row storage and new column storage. For the same data and table, Oracle in-memory database can simultaneously store them in both formats, and at the same time update (add/delete/alert) and keep consistent. Such as adding a row to a table, the data stored in column format will also update synchronously. It plays the same role in the performance improvement of OLAP, removes a large number of indexes used in statistic, and indirectly promotes the performance of OLTP.

Memory database is significant different from traditional database in many implementation technologies ${ }^{[3]}$. These differences are reflected in index technology, concurrency control and so on. System reads data from disk, and the operation bottleneck usually comes from the I/O operation from disk to memory, especially for the operation application involving a large amount of data access ${ }^{[4,5]}$. In order to improve the processing speed of system, we can consider enhancing the level of software and hardware. For software, we can adopt good memory data management technology, realize the full potential of large memory, and reduce the I/O operations from disk to memory, which can significantly improve the processing speed of system. For hardware and network, it contains the promotion of data communication speed and GPU/FPGA computing acceleration.

\section{Infrastructure Oriented OLAP and OLTP Application.}

With the development of open source technology, more and more users tend to adopt more open source components in consideration of cost and commercial implementation, which is a challenge of system design. This work is aimed at the hybrid application of OLAP and OLTP, and we propose an architecture solution based on x86 server and open source components, which is shown in Fig.1.

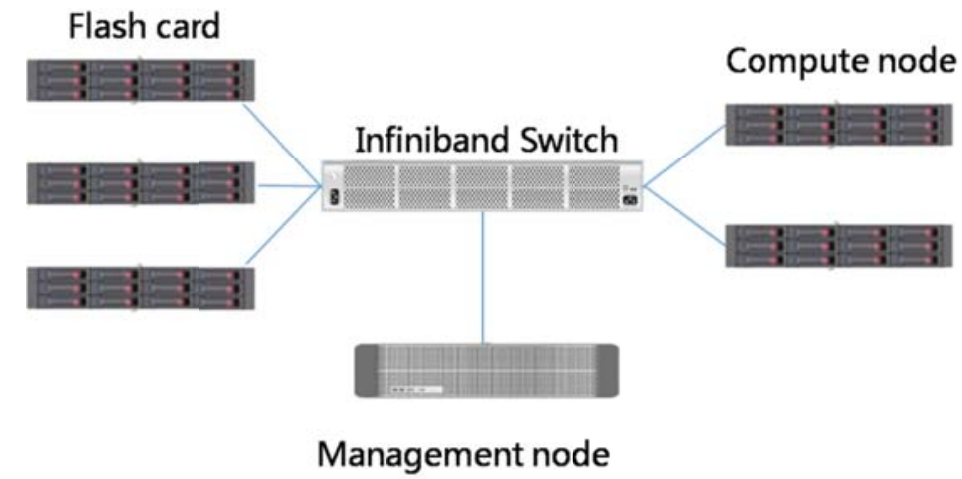

Fig. 1. Hybrid application architecture of oriented OLAP and OLTP

Database and Hadoop can be loaded to the computational node ${ }^{[6]}$. Infiniband switch provides high I/O speed and we can improve data access speed by using RDMA if we choose Oracle database. Data is carried by the server installed Flash card and we can achieve Raid5 by clustering technology. For now, Flash card has TB level capacity and GB level read-write speed, which fills the gap between memory and disk and then forms an effective complement with large-scale memory computing.

We choose $\mathrm{x} 86$ server in large applications considering not only processing power but also the factor of sever reliability. The reliability of Unix server is better than $\mathrm{x} 86$ server according to the reliability investigation report of global server hardware and server operating system published by ITIC (Information Technology Industry Council) and the downtime of each enterprise device each year. When a Unix application is moved to x 86 server, we must ensure that the reliability can't be decreased. So we need configure appropriate number of $\mathrm{x} 86$ servers to achieve the same reliability with Unix servers. The formula is as follows:

$$
\left(1-P_{U N I X}\right)^{X}=\left(1-P_{\text {LINUX }}\right)^{Y}
$$

Take IBM AIX (with the reliability of $99.997 \%$ ) and RED HAT (with the reliability of $99.987 \%$ ) for examples, when $X=2$ and $Y=2.5$, the two sides are equal. Therefore, the entire reliability can 
be improved by uniformly increasing the number of $x 86$ servers. In this work, the number of servers with Flash card is 3 at least and can be extended linearly.

\section{Memory Computation Acceleration.}

This work involves memory computation acceleration technology, which includes high speed interconnection based on PCI-E and computation acceleration based on FPGA.

High Speed Interconnection Based on PCI-E. The bandwidth of PCI-E 3.0 can reach 10GB/s and the signal frequency of it increases from $5 \mathrm{GT} / \mathrm{s}$ of PCI-E 2.0 to $8 \mathrm{GT} / \mathrm{s}$. PCI-E 3.0 achieves point-to-point data exchange under the condition of no CPU auxiliary. In the full-duplex mode, the throughput of twelve ports can achieve $256 \mathrm{Gbps}$ and these ports are packaged by TCP/IP protocol interface for expending application.

The advantage of using PCI-E to data exchange is that all peripherals can be mapped to local server. Thus the peripherals can remotely access the memory or high speed FLASH card of different servers. Then the system implements functions like RDMA and can work as an Infiniband switch.



Fig. 2. Data acceleration by PCI-E

Computation Acceleration Based on FPGA. Both GPU and FPGA are common algorithm acceleration technologies ${ }^{[7,8]}$. In terms of current technology development, the comparisons between GPU and FPGA are as follows:

- GPU: strong parallel computing ability. And the use of high-level language can make developers focus on the research and implementation of algorithm. The disadvantage is high energy consumption and not fit for large scale use.

- FPGA: strong parallel computing ability. The technology implementation is difficult and each program statement presents actual hardware module. The advantage is low energy consumption and conducive to large scale use.

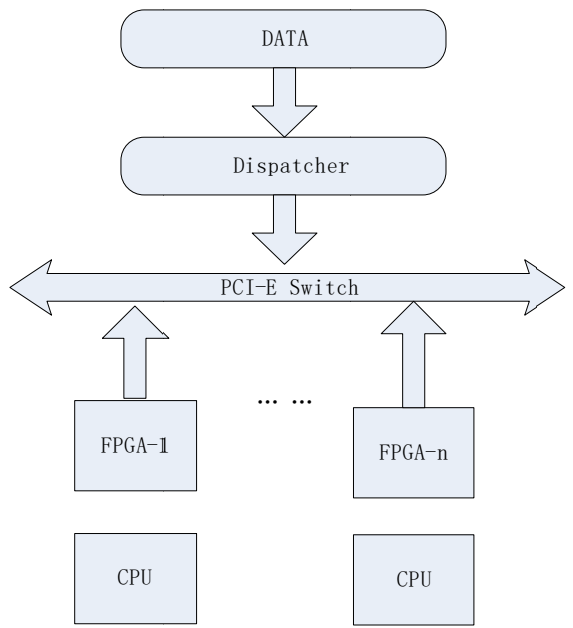

Fig. 3. Realization of data acceleration adopting FPGA

Hence we can see that GPU is suitable for processing complex algorithms. FPGA can also implement complex algorithms since DSP and ARM module are added to the high-end devices of 
FPGA. Although the power meter pricing data in this work is large, the algorithm is relatively simple, so we use FPGA acceleration. Because pricing formula is not the same for users, we need to consider both task allocation and parallel computing method. Furthermore, there exits 20-80 rule in power meter pricing, that is, $80 \%$ calculations use $20 \%$ pricing formulas. So we can consider implementing the pricing formulas with more than 100,000 users by FPGA.

\section{Conclusion.}

In terms of traditional IT infrastructure, owing to the not open feature and free of compatibility, the development of information is increasingly restricted, and open architecture becomes an inevitable trend. In recent years, the Internet and related market in information field have changed greatly, and some large companies have been booming while some started to fade, which presents the increasingly obvious mutual promoted effects between technology and market. With the progress of technology, the threshold of software and hardware technology gradually gets reduced, which contributes to the emergence of a set of companies that define software and hardware products by service. The birth and development process of them are capable of bringing enlightenment to the whole information industry. Depending on the business advantages, it becomes a trend that an integration of business, software and services drives the development of software and hardware industrial chains. This work is based on this developing trend, the future work will focus on further improving PCI-E switch speed andpromoting usability of EPGA.

\section{References}

[1] Hector Garcia Molina, Kenneth Salem. Main Memoy Database Systems: An Overview. IEEE Transactions on Knowledge and Data Engineering, Vol 4, No 6, (1992)

[2] K. Salem and H. Garcia-Molina. "System M: A transaction processing tested for memory resident data". IEEE Transactions on Knowladge and Data Engineering, Vol 2,No1, (1990), pp.161-172

[3] H.V. Jagadish, Daniel Lieuwen, Rajeev Rastogi, Avi Silberschatz. "Dali: A high performance main memory storage manager", Proceedings of the 20th VLDB conference,(1994)

[4] Ted Lewis ,Hesham El-Rewini, Introduction to parallel computing。 Prentice-Hall, Inc.(1992)

[5] F Chang.Bigtable:Adistributedstoragesystemfor structured data. ACM Transactions on Computer Systems, Vol 26,No2,(2006),pp.205--218

[6] D Singh, CK Reddy.A survey on platforms for big data analytics. Springer 《Journal of Big Data》,(2014), pp.1-20

[7] M News.Storage System for 'Big Data' Speeds Access to Information 《Communications of the Acm》, (2014)

[8] C Fang.Using NVMe Gen3 PCIe SSD Cards in High-density Servers for High-performance Big Data Transfer Over Multiple Network Channels, 《Slac Document Server》, (2015) 